\title{
AN INVESTIGATION OF OPTICAL VIBRATIONS IN $\mathrm{Zn}_{3} \mathrm{P}_{2}{ }^{*}$
}

\section{J. MisiewiCz,}

Institute of Physics, Technical University of Wrocław, Wybrzezie Wyspianiskiego 27, 50-370 Wrocław, Poland

\section{J.M. Wrobel ${ }^{\dagger}$ and B.P. Clayman}

Department of Physics, Simon Fraser University, Burnaby, B.C. V5A1S6, Canada

(Received August 8, 1990)

\begin{abstract}
Reflectivity and transmittivity spectra of $\mathrm{Zn}_{3} \mathrm{P}_{2}$ in the far infrared region were measured at several temperatures. Raman scattering spectra at $295 \mathrm{~K}$ were also measured. Results of these measurements were interpreted in terms of one-phonon and multi-phonon transitions.
\end{abstract}

PACS numbers: 63.20.-e, 78.30.Hv

\section{Introduction}

$\mathrm{Zn}_{3} \mathrm{P}_{2}$ is relatively little known but interesting compound due to both its application and its basic properties. Till now, only a few papers have been published in which the lattice modes of $\mathrm{Zn}_{3} \mathrm{P}_{2}$ were investigated [1-5].

TABLE I

Zone center infrared active phonons in $\mathrm{Zn}_{3} \mathrm{P}_{2}\left(\right.$ in $\left.\mathrm{cm}^{-1}\right)$.

\begin{tabular}{l|c|c|c|c|c|c|c|c}
\hline \hline \multicolumn{10}{c}{ Temperature 295 K } \\
\hline TO & 44 & 64 & 70.5 & 78 & 86 & 103 & 168.5 & \\
LO & 57 & 66 & 71 & 78.5 & 89 & 104.5 & 169 & \\
\hline TO & 182.5 & 246 & 285 & 309.5 & 338 & 351 & & \\
LO & 184 & 276 & 307 & 320 & 345 & 360 & & \\
\hline \multicolumn{8}{c}{ Temperature 10 K } \\
\hline TO & 46.5 & 62 & 71 & 77 & 87.5 & 104 & 110.5 & 170.5 \\
LO & 58.5 & 63.7 & 71.5 & 78 & 94 & 105.5 & 112.5 & 172 \\
\hline TO & 184 & 243 & 285 & 301.5 & 312.5 & 331.5 & 340 & 354 \\
LO & 186 & 274 & 309.5 & 301.5 & 323 & 333 & 348.5 & 363
\end{tabular}

${ }^{*}$ This work was supported in part by CPBP 01.04.I.2.7 and by a grant from the Natural Sciences and Engineering Council of Canada.

tDepartment of Physics, University of Missouri, Kansas City, M064110, USA. 
$\mathrm{Zn}_{3} \mathrm{P}_{2}$ crystal structures is tetragonal and described by space group $D_{4 h}^{15}[6]$. The unit cell is composed of 40 atoms producing 120 phonon branches, 117 optical and 3 acoustic modes. Optical measurements were performed on high-resistivity, low concentration samples $\left(p<10^{15} \mathrm{~cm}^{-1}\right)$. Details of the experimental techniques as well as sample preparation method are presented elsewhere [4-5].

\section{Results and discussion}

Figure 1(a) presents reflectivity spectra in the reststrahlen region. As expected, a complicated structure is observed. In the first region $\left(40-115 \mathrm{~cm}^{-1}\right)$ six peaks are visible at room temperature; their structure becomes clearer at low temperature. A relatively broad peak around $100 \mathrm{~cm}^{-1}$ splits into two independent ones. Very prominent features are located within $240-360 \mathrm{~cm}^{-1}$. Five maxima are visible at $295 \mathrm{~K}$ and seven more sharpened at low temperature. Between these two ranges a relatively flat plot in the $150-180 \mathrm{~cm}^{-1}$ energy range can be found. Lowering the temperature causes two distinct maxima to appear.

TABLE II

Infrared overtones at $\Gamma$ calculated from the data of Table I compared with transitions observed in VV Raman scattering (in $\mathrm{cm}^{-1}$ ).

\begin{tabular}{l|c|c|c|c|c|c|c|c|c}
\hline \hline $\begin{array}{l}\text { IR } \\
\text { calculated }\end{array}$ & 114 & 122 & 142 & 157 & 178 & 209 & 338 & 368 & \\
\hline $\begin{array}{l}\text { Raman } \\
\text { observed }\end{array}$ & 86.5 & 125 & 144 & 159 & 171 & 212 & 340 & 363 & 499
\end{tabular}

Group theory analysis gives the representation of infrared active one-phonon modes as $\Gamma^{\mathrm{IR}}=9 \Gamma_{2}^{-}+15 \Gamma_{5}^{-}$, so we should expect 9 single one-phonon and 15 double degenerated ones as infrared active. Performing Kramers-Kronig analysis of reflectivity spectra we determined energies of TO and LO infinite wavelength phonons (Table I). To explain the number of observed experimentally one-phonons, it is necessary to assume that some of theoretically degenerated modes $\left(\Gamma_{5}^{-}\right)$are split.

Transmittance spectra at several temperatures are presented in Fig. 1(b). From the temperature dependence of transmittance spectra we may expect that transitions located at approx. 500,525, 560,590,625,640,660,690 $\mathrm{cm}^{-1}$ are connected with sums of one-phonon transitions. A more detailed analysis of the temperature dependence of reflectivity and absorption spectra will be published [7]. An analysis of the Raman tensor shows that in the VV configuration $9 \Gamma_{1}^{+}$and $10 \Gamma_{3}^{+}$ one-phonons should be visible and $16 \Gamma_{5}^{+}$doubly degenerated ones at VH configuration. Figure 2 presents Raman scattering spectra at room temperature. More prominent features, denoted in this figure by arrows, we connect with one-phonon transitions. The weaker ones we explain as multiphonon transitions.

Similarly to reflectivity data, it seems to be necessary to assume that some of theoretically degenerated modes $\left(\Gamma_{5}^{+}\right)$are split. $\mathrm{Zn}_{3} \mathrm{P}_{2}$ crystal possesses inversion symmetry, so due to mutual exclusion rule the same transitions should not be observed in infrared and Raman spectra. Almost all, from the total number 


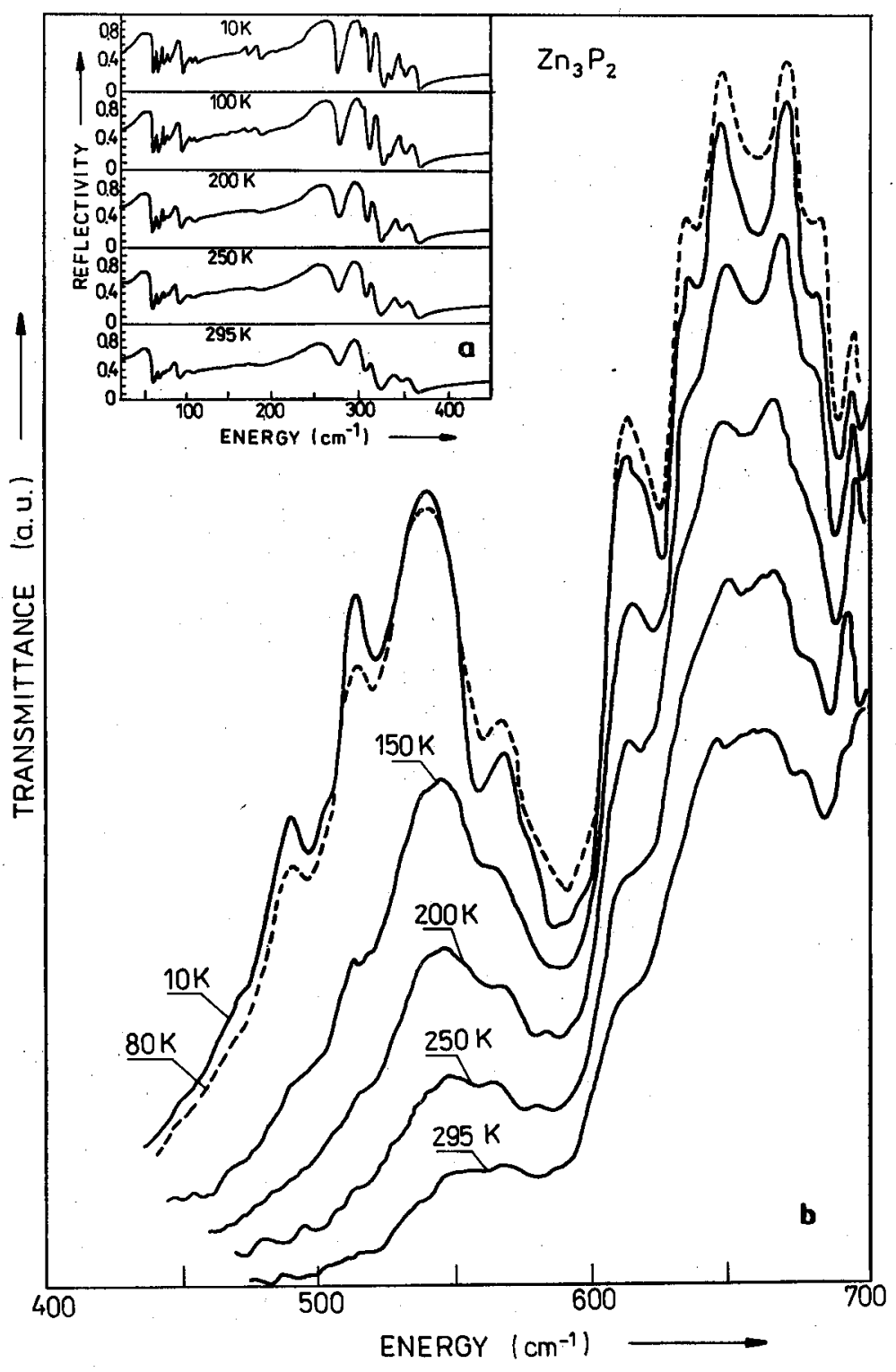

Fig. 1. (a) Reflectivity spectra of $\mathrm{Zn}_{3} \mathrm{P}_{2}$ within reststrahlen region as a function of temperature; (b) transmittance spectra of $\mathrm{Zn}_{3} \mathrm{P}_{2}$ at several temperatures. 


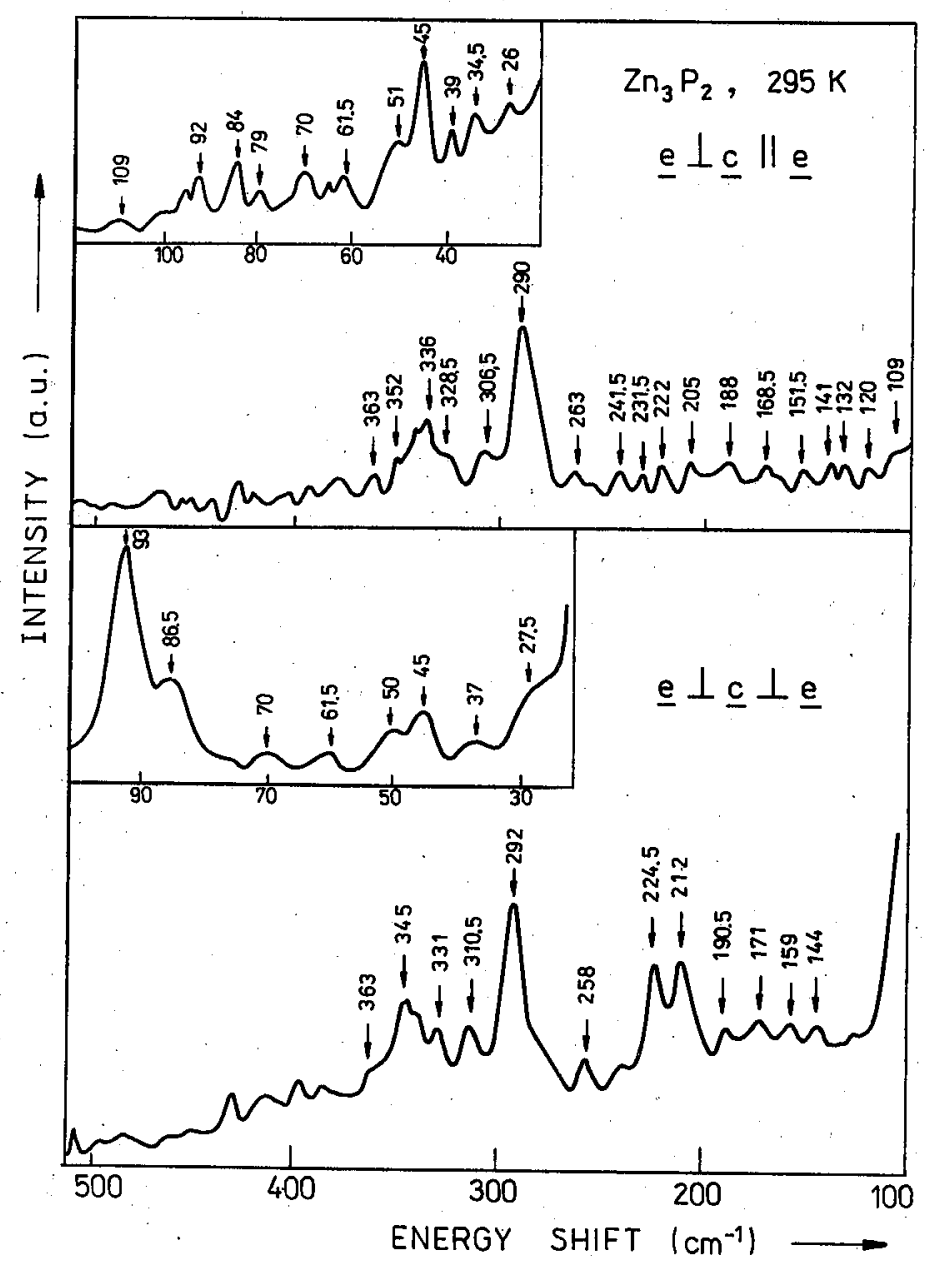

Fig. 2. Raman scattering spectra of $\mathrm{Zn}_{3} \mathrm{P}_{2}$ at room temperature in two configurations: $e \perp c \| e(\mathrm{VH})$ and $e \perp c \perp e(\mathrm{VV})$; arrows denote energies of one-phonon transitions. 
of one-phonon energies observed in Raman scattering and determined from reflectivity spectra ones are different (compare the data from Tab. I and Fig. 2), so this rule seems to be well satisfied. Group theory predicts no overtones of infrared active phonons at $\Gamma$ allowed in infrared but they should be Raman active in VV configuration (Table II).

Understanding of the phonon spectra in $\mathrm{Zn}_{3} \mathrm{P}_{2}$ needs more investigations e.g. temperature dependence of Raman scattering, detailed study of multiphonon absorption as a function of temperature as well as neutron scattering experiments.

\section{Acknowledgments}

The authors are indebted to Dr D. Heiman, of the Francis Bitter National Magnet Lab at MIT (USA), who made it possible to perform the Raman scattering measurements.

\section{References}

[1] R.S. Radautsan, N.N. Syrbu, J.J. Nebola, V.J. Volodina, Sov. Phys. Solid State 19, 1290 (1977).

[2] J. Misiewicz, B. Sujak-Cyrul, A. Bartczak, Solid State Commun. 58, 677 (1986).

[3] J. Misiewicz, J.M. Wrobel, B.P. Clayman, F. Królicki, in Proc. 16th School Phys. Semicond. Comp. Jaszowiec 1987, Acta Phys. Pol. A73, 407 (1988).

[4] J. Misiewicz, J.M. Wrobel, B.P. Clayman, Solid State Commun. 66, 747 (1988).

[5] J. Misiewicz, J. Phys. Condens. Matter 1, 9283 (1989).

[6] M.V. Stackelberg, P. Paulus, Z. Phys. Chem. 28B, 427 (1935).

[7] J. Misiewicz, J.M. Wrobel, B.P. Clayman, to be published. 